\title{
Control of an industrial polymerization reactor using flatness
}

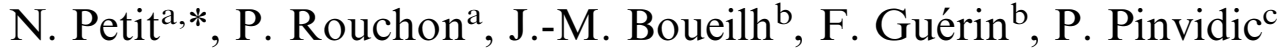 \\ ${ }^{a}$ Centre Automatique et Systèmes, École Nationale Supèrieure des Mines de Paris, 60, boulevard Saint-Michel, 75272 Paris Cedex 06, France \\ ${ }^{\mathrm{b}}$ Centre Technique ATOFINA, Chemin de la Lône BP 32, 69492 Pierre Bénite Cedex. France \\ ${ }^{\mathrm{c}} A P P R Y L$, Usine PP2, BP 21, 13117 Lavéra, France
}

Received 8 November 2000; received in revised form 26 May 2001; accepted 21 June 2001

\begin{abstract}
The aim of this paper is to report the design and use of a controller for the world's largest polypropylene reactor. This is the first industrial process-controller to use the so-called flatness property of the system, which is presented here in a concise and application oriented manner. Industrial results are given and the control strategy is presented in the context of today's fast and competitive market of polymers. (C) 2002 Elsevier Science Ltd. All rights reserved.
\end{abstract}

Keywords: Polypropylene; Continuous stirred tank reactor; Control; Flatness; Industrial application

\section{Introduction}

The aim of this paper is to report the design and use of a unique controller in an industrial framework. This controller is worth mentioning because it is the first application of flatness in industrial process control and also because the system under consideration is the largest propylene polymerization plant in the world [15].

Originally studied for mechanical systems $[3,4]$, flatness exposes important issues in nonlinear control theory such as interpretations of controllability and feedback linearization $[4,5,7,11]$. Flatness implies a oneto-one correspondence between the trajectories of the system and those of a reduced set of variables called flat outputs. Many of the problems that are known to be very difficult to solve for nonlinear systems such as trajectory generation and tracking are thus transposed into a lower dimensional space, where they become straightforward. This is the methodology followed in this paper.

Two quantities are of particular interest when producing polypropylene in this plant located in Lavera (south of France): the amount of production and the melt-index of the polymer. The melt-index indicates some of the mechanical properties of the polymer and is critical for injection and thermoforming transforma-

\footnotetext{
* Corresponding author. Tel.: +33-1-40-51-93-29; fax: +33-1-4051-91-65.

E-mail address: petit@cas.ensmp.fr (N. Petit).
}

tions [2] (see also the http://www.appryl.fr). These two quantities depend in a nonlinear way on the amount of catalyst and hydrogen that are present in the reactor. The amount of production and the melt-index are planned with respect to economical considerations (i.e. the market of polymers). This induces frequent changes in the setpoints that must be met fast and with precision to optimize profit. This critical issue arises in different polymerization processes, see for instance [9] and [16].

Thus, the main challenge is to control the system for a wide range of setpoints with high accuracy and dynamical performance. These requirements suggest that controllers based on linear approximations of the system are unlikely to be very successful.

This system is very complex. Precise simulations models of this continuous stirred tank reactor involve thousands of variables. Yet, for control purpose, we concentrate on a reduced set of 4 differential equations and 2 nonlinear mappings, originating from balance equations and statistical studies. From a mathematical point of view, this can be seen as a two input two output model with a delay on one input. This model is both compact and rich enough to represent with accuracy the behaviour of the reactor.

The controller designed here takes into account these nonlinearities and the delay. It is capable of doing fast and precise transients, fulfilling the requirements above.

The paper is organized as follows. In Section 1 we give a model of the process. In Section 2 we recall the 


\section{Nomenclature}

$Q_{a} \quad$ (in $\mathrm{kg}$ ) is the amount of catalyst in the reactor

$X \quad$ (dimensionless) is the rate of solid $(0 \leqslant X \leqslant 1)$ (ratio between the mass of solid and the mass of solid + the mass of liquid particles)

Prod (in $\mathrm{kg} \mathrm{s}^{-1}$ ) is the instantaneous amount of produced polymer

$C_{\mathrm{H}_{2}} \quad\left(\mathrm{in} \mathrm{mol} \mathrm{m} \mathrm{m}^{-3}\right.$ ) is the hydrogen concentration

MI (dimensionless) is the melt-index of the polymer in the reactor

$u \quad\left(\right.$ in $\left.\mathrm{kg} \mathrm{s}^{-1}\right)$ is the amount of catalyst coming in the reactor per unit of time

$v \quad$ (in mol m $\mathrm{m}^{-3} \mathrm{~s}^{-1}$ ) is the amount of hydrogen coming in the reactor per unit of time per unit of volume

$\tau \quad$ (in s) is the residence time. It is a constant.

definition of the flatness property and show that this model is flat. We use this property to design control strategies. In Section 3, we present industrial results of our controller and discuss comparisons with other possible approaches. In conclusion we underline the tradeoff between the difficulty of building up a relevant nonlinear model and the simplicity of the resulting controller.

\section{Modeling}

The polymerization process is depicted in Fig. 1. The hydrogen enters the reactor directly while the catalyst enters the reactor after a delay due to activation preprocessing. Roughly speaking, the catalyst acts upon the amount of production, while the hydrogen acts upon the melt-index of the polymer. using the following nomenclature it is possible to write a nonlinear model.

\subsection{Model}

The process depicted in Fig. 1 can be represented by

$\frac{\mathrm{d}}{\mathrm{d} t}\left(Q_{a}\right)=u(t-\delta)-\frac{Q_{a}}{\tau}$

$\frac{\mathrm{d}}{\mathrm{d} t}(X)=Q_{a}(\alpha X+\beta)-\gamma X+\xi \frac{X}{1-X}$

$y_{1}=\operatorname{Prod}=\varphi \frac{X}{1-X}$

$\frac{\mathrm{d}}{\mathrm{d} t}\left(C_{H 2}\right)=v-g\left(C_{\mathrm{H}_{2}}, Q_{a}\right)$

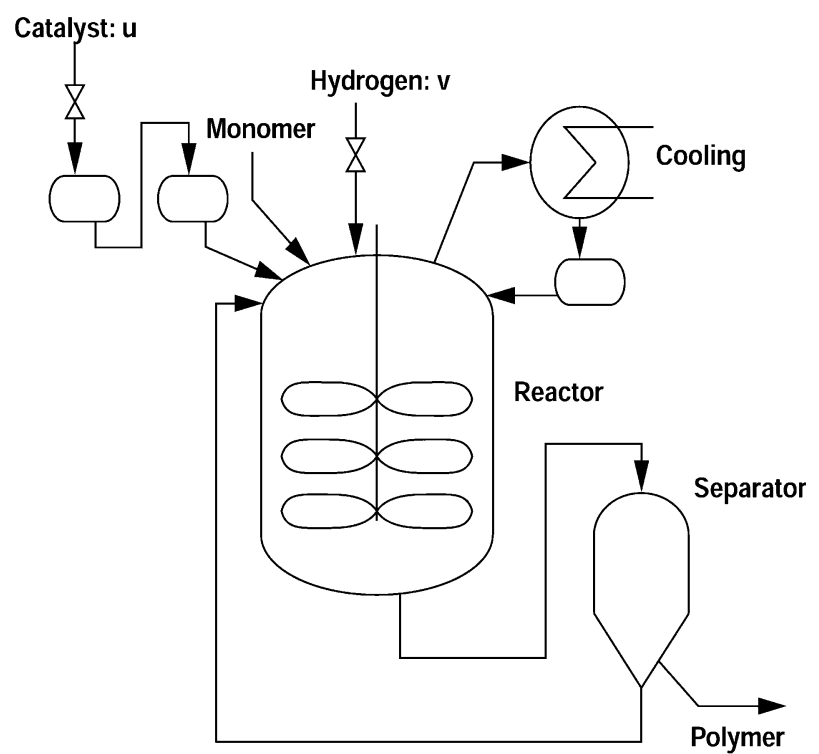

Fig. 1. The polymerization process: 2 inputs $(u, v), 2$ outputs (meltindex and amount of production).

$\frac{\mathrm{d}}{\mathrm{d} t}(\log M I)=\frac{a \log C_{\mathrm{H}_{2}}+b-\log M I}{\tau}$

$y_{2}=M I$

where $a, b$ are constant dimensionless coefficients, $\alpha, \beta$ (both in $\mathrm{s}^{-1} \mathrm{~kg}^{-1}$ ) and $\gamma, \xi$, (both in $\mathrm{s}^{-1}$ ) and $\varphi$ (in $\mathrm{kg}$ $\mathrm{s}^{-1}$ ) are combinations of densities and other known operating parameters (omitted here for sake of clarity), $\delta$ (in s) is a constant delay. As mentioned before the effect of $u$ is primarily on the amount of production. Still, one can clearly see the interaction of $u$ on $M I$ that appears through $g\left(C_{\mathrm{H}_{2}}, Q_{a}\right)$. Finally, the residence time $\tau$ is assumed to be a constant thanks to a low level regulatory loop acting upon the level of the reactor.

Eq. (1) is a dilution equation with a constant delay $\delta$ on the input. Eq. (2) is a mass balance equation. Eq. (4) is a balance equation and includes a nonlinear inference. Eq. (5) is a mixing equation where the source term arises from theoretical chemical studies of polymer growth, (see [8] for a similar study).

This model captures the essential elements of the dynamics. It is quite precise: we represent in Fig. 2 a comparison between real-time measurements of the production Prod and simulation results obtained with this model. These results were obtained for a one day period and are representative.

\section{Flatness of the model}

The flatness property of a (nonlinear) dynamical system $\dot{x}=f(x, u)$ with $x \in \mathbb{R}^{n}, u \in \mathbb{R}^{m},(n, m) \in \mathbb{N}$ is described as follows $[3,4]$ 


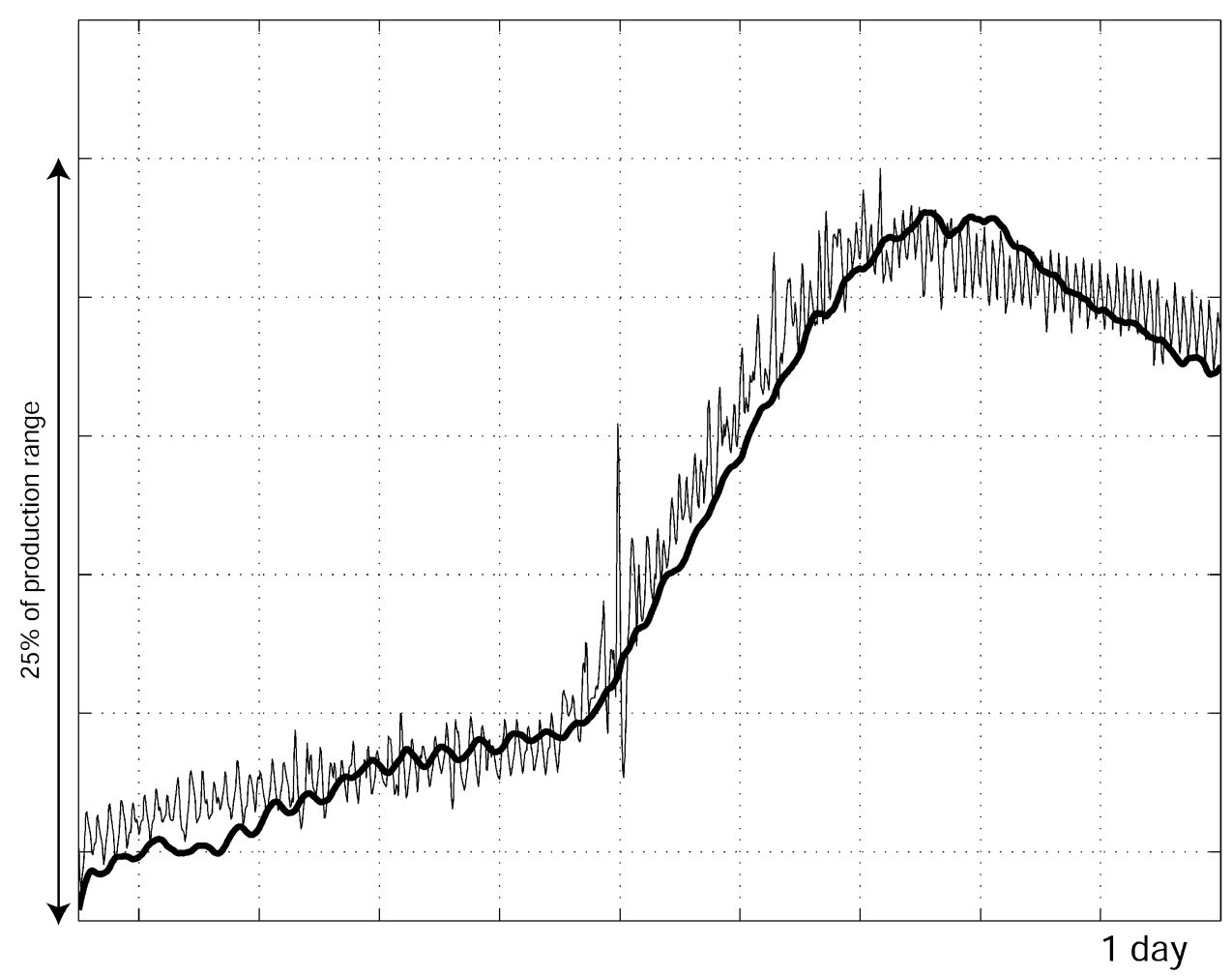

Fig. 2. Accuracy of the model. Comparison between real-time measurements of the production (Prod) and simulation results obtained with the model $(1,2,3,4,5,6)$. Time period $=1$ day.

Definition 1. ([3,4]). The system $\dot{x}=f(x, u), y=h(x)$ with $x \in \mathbb{R}^{n}, u \in \mathbb{R}^{m},(n, m) \in \mathbb{N}$, is flat if and only if there exists a variable $z$ called the flat output such that

$$
\begin{aligned}
& x=A\left(z, \dot{z}, \ldots z^{(n-1)}\right) \\
& y=B\left(z, \dot{z}, \ldots z^{(n-1)}\right) \\
& u=C\left(z, \dot{z}, \ldots z^{(n)}\right)
\end{aligned}
$$

where $A, B$ and $C$ are three mappings (depending on $f$ and $h$ ), and $z^{(i)}$ denotes the $i$ th derivative of the output $z .{ }^{1}$

In the previous definition the equations mean that there exists a quantity $z$ that summarizes the behaviour of the whole system via the mappings $A$ and $B$. The trajectories of the system, i.e. $(x, u)$, are easily computed by the trajectories of $z$ and its derivatives without integrating any differential equation.

To see how this property appears in our particular problem, one may write the previous equations in this form

$$
\begin{aligned}
& \dot{x}_{1}=u(t-\delta)-\frac{x_{1}}{\tau} \\
& \dot{x}_{2}=x_{1} f\left(x_{2}\right)+h\left(x_{2}\right)
\end{aligned}
$$

\footnotetext{
1 This definition is very general. In particular the order of the flat output need not be $n$ in the multi input multi output case.
}

$$
\begin{aligned}
& \dot{x}_{3}=v-g\left(x_{3}, x_{1}\right) \\
& \dot{x}_{4}=\frac{a \log \left(x_{3}\right)+b-x_{4}}{\tau} \\
& y_{1}=k\left(x_{2}\right) \\
& y_{2}=\exp \left(x_{4}\right)
\end{aligned}
$$

where $f$ is a strictly positive function (on its interval of definition $[0,1])$. It is easy to see that this system is flat: ${ }^{2}$ all the variables are parameterized by the flat outputs $x_{2}=X, x_{4}=M I$ and their derivatives.

More precisely

$x_{3}=\exp \left(\frac{\tau \dot{x}_{4}+x_{4}-b}{a}\right)$

$x_{1}=\frac{\dot{x}_{2}-h\left(x_{2}\right)}{f\left(x_{2}\right)}$

$y_{1}=k\left(x_{2}\right)$

$y_{2}=\exp \left(x_{4}\right)$

${ }^{2} \delta$-Flatness is the precise definition. This notion introduced in [11], see also [12], addresses the particular case of delay systems. 
and

$$
\begin{aligned}
u(t-\delta)= & \frac{\ddot{x}_{2}-\dot{x}_{2} h^{\prime}\left(x_{2}\right)}{f\left(x_{2}\right)}-\left(\dot{x}_{2}-h\left(x_{2}\right)\right) \frac{\dot{x}_{2} f^{\prime}\left(x_{2}\right)}{f^{2}\left(x_{2}\right)} \\
& +\frac{\dot{x}_{2}-h\left(x_{2}\right)}{\tau f\left(x_{2}\right)} \\
v= & \exp \left(\frac{\dot{x}_{4} \tau-b+x_{4}}{a}\right) \frac{\ddot{x}_{4} \tau+\dot{x}_{4}}{a} \\
& +g\left(x_{4}, \dot{x}_{4}, x_{2}, \dot{x}_{2}\right) .
\end{aligned}
$$

\subsection{Open-loop control strategy}

A general property of flat systems $[3,4]$ is that it suffices to control the flat outputs to control the whole system. In our case once $x_{2}$ and $x_{4}$ are controlled, so are $x_{3}, x_{1}, y_{1}, y_{2}$ because of Eqs. (13)-(16). The open loop controls are given by Eqs. (17) and (18).

Example. We detail here an example of an open-loop control calculation. Assume that the operator wishes to increase the setpoint for the amount of production from $\operatorname{Prod}_{\text {initial }}$ to Prod ${ }_{\text {objective while keeping the melt-index }}$ $M I_{\text {initial }}$ constant.

The trajectory of the amount of production is transformed to the flat outputs: $x_{2}$ must go from $X_{\text {initial }}$ to $X_{\text {objective where }}$

$$
\begin{gathered}
\operatorname{Prod}_{\text {initial }}=\varphi\left(\frac{X_{\text {initial }}}{1-X_{\text {initial }}}\right) \\
\operatorname{Prod}_{\text {objective }}=\varphi\left(\frac{X_{\text {objective }}}{1-X_{\text {objective }}}\right),
\end{gathered}
$$

while $x_{4}$ will remain constant. A transition in finite time $T$ between $X_{\text {initial }}$ and $X_{\text {objective }}$ is prescribed by any function joining these two setpoints, e.g. a polynomial, denoted by $[0, T] \ni t \mapsto x_{2}^{\text {ref }}(t)$. Then the open-loop control is computed via (17) and (18) as

$$
\begin{aligned}
& u^{o l}(t)=\frac{\ddot{x}_{2}^{\mathrm{ref}}(t+\delta)-\dot{x}_{2}^{\mathrm{ref}}(t+\delta) h^{\prime}\left(x_{2}^{\mathrm{ref}}(t+\delta)\right)}{f\left(x_{2}^{\mathrm{ref}}(t+\delta)\right)} \\
& -\left(\dot{x}_{2}^{\mathrm{ref}}(t+\delta)-h\left(x_{2}^{\mathrm{ref}}(t+\delta)\right)\right) \frac{\dot{x}_{2}^{\mathrm{ref}}(t+\delta) f^{\prime}\left(x_{2}^{\mathrm{ref}}(t+\delta)\right)}{f^{2}\left(x_{2}^{\mathrm{ref}}(t+\delta)\right)} \\
& +\frac{\dot{x}_{2}^{\mathrm{ref}}(t+\delta)-h\left(x_{2}^{\mathrm{ref}}(t+\delta)\right)}{\tau f\left(x_{2}^{\mathrm{ref}}(t+\delta)\right)} \\
& v^{o l}(t)=g\left(\log \left(M I_{\text {initial }}\right), 0, x_{2}(t), \dot{x}_{2}(t)\right) .
\end{aligned}
$$

In Fig. 3 one can see an example of such a calculation. Given a polynomial transition function for the flat output $x_{2}$ we compute the control $u$ via (17). One can

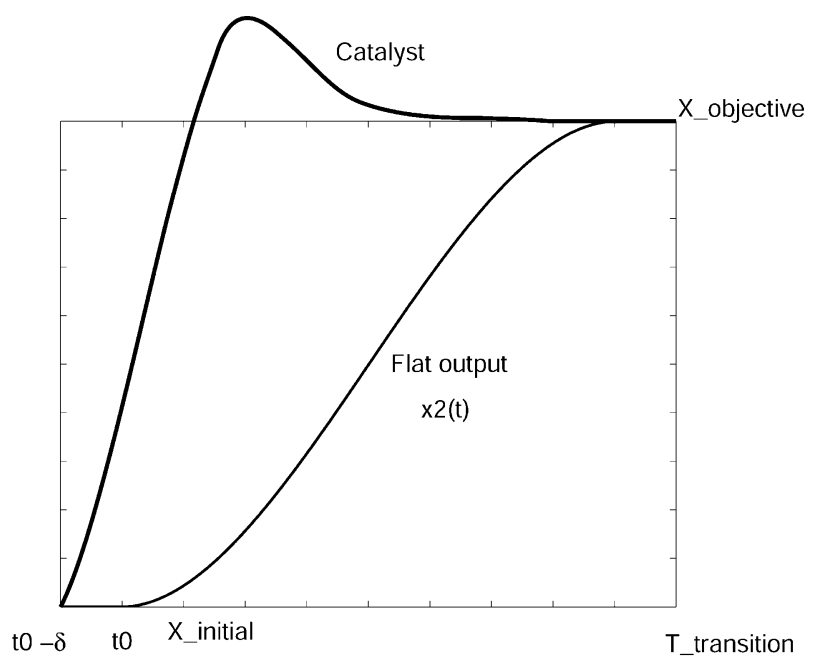

Fig. 3. Open-loop control strategy. The operator's request is expressed in terms of a transition for the flat output $X$ and the open-loop control is computed via (17).

clearly see the effect of the advance in formula (19): the control starts increasing before one can expect the flat output to increase (with exactly a $\delta$ advance). The (input) overshoot occurs while the output is still far from the setpoints.

\subsection{Closing the loop}

In fact the open-loop strategy must be complemented by a feedback control law.

As mentioned before, once the flat outputs are stabilized, the whole system is stabilized because all the variables of the system are expressed in terms of the flat outputs via Eqs. (13)-(16).

The dynamics of the flat outputs are given by (17) and (18) We can stabilize them. To satisfy the following stable closed loop equations for the flat output

$$
\begin{aligned}
& \left(\ddot{x}_{2}-\ddot{x}_{2}^{\text {ref }}\right)=-k_{1}\left(\dot{x}_{2}-\dot{x}_{2}^{\text {ref }}\right)-k_{2}\left(x_{2}-x_{2}^{\text {ref }}\right) \\
& \left(\ddot{x}_{4}-\ddot{x}_{4}^{\text {ref }}\right)=-k_{3}\left(\dot{x}_{4}-\dot{x}_{4}^{\text {ref }}\right)-k_{4}\left(x_{4}-x_{4}^{\text {ref }}\right)
\end{aligned}
$$

where $k_{1}, k_{2}, k_{3}, k_{4}$ are constants, it suffices to substitute these desired $\ddot{x}_{2}$ and $\ddot{x}_{4}$ in Eqs. (17) and (18). This gives the closed loop controller

$$
\begin{aligned}
& u(t-\delta)=u^{o l}(t-\delta)+h_{1}\left(x_{2}-x_{2}^{\mathrm{ref}}, \dot{x}_{2}-\dot{x}_{2}^{\mathrm{ref}}, x_{2}, \dot{x}_{2}\right) \\
& v=v^{o l}+h_{2}\left(x_{4}-x_{4}^{\mathrm{ref}}, \dot{x}_{4}-\dot{x}_{4}^{\mathrm{ref}}, x_{4}, \dot{x}_{4}\right) .
\end{aligned}
$$

Some required variables in Eqs. (20) and (21) are not available: $x_{2}$ is not measured and $x_{4}$ is measured at discrete times (with a delay due to the necessary laboratory analysis). To overcome this we use estimators based on 
classical least-squares methods, predictors, and Luenberger-style observer. These observers give naturally stable dynamics that do not interfere with the stability of the closed-loop controller. The derivatives were approximated by passive low-pass filters.

\section{Industrial results}

Our controller has been in full service since July 1999 and allows optimization of profit. As one can see in Fig. 4, the controller allows very fast and precise transients. On the same figure one can clearly see the effect of the delay compensation by a "time advance" in the controller design [see Eq. (19)]. Before the system meets the setpoints, the controller stops changing the value of the input (catalyst), preventing the overshoot in the production rate. These results are representative of the overall behavior of our controller.

We give industrial results for melt-index transitions on Fig. 5. The controller is capable of simultaneous transitions for the amount of production Prod and for the melt-index $M I$.

\subsection{Comparisons with other techniques}

Of course it is not possible to compare these results with every possible controller. Yet, we tried to tune some basic linear controllers (PI and LQR) for various simulations. Unfortunately we did not experience good results on the real plant when dealing with large changes in the setpoints and then decided to shift to another solution (the flatness approach presented here): it was difficult to combine good dynamical performances and robustness to perturbations. It is possible to sketch that with a linear controller the system may take about twice as long to converge as with the flatness controller, and that the overshoots would be very difficult to prevent without any serious deterioration of the dynamical performances. We represent in Fig. 6 a comparison between such a LQR controller acting on a simulator (using the model presented before under similar conditions, i.e. using real data for the coefficients $\gamma, \xi$ and $\varphi$ ) and the real-time results of our controller on the plant.

Another question of relevance is: how does it compare to the well established model predictive controller (MPC) approach (see again [9] for instance)? First it should be noted that here the control objective does not really express in terms of a well-defined cost function: the main goal is to get transitions as repeatable as possible. In other terms, provided that the starting and ending setpoints are the same, a transition should always take the same time and be as accurate. Yet, as usual with flat systems, see again $[3,4]$, the flatness Eqs, namely (13)-(18) express all the trajectories of the system. Should the control objective be expressed as a cost, it would have been possible, and computationally profitable as it has been pointed out in other applications $[1,6,10,14]$, to solve the optimal control problem through these flatness equations. The unknown would be the shapes of the transition functions for the flat outputs $[0, T] \ni t \mapsto x_{2}^{\mathrm{ref}}(t)$ and $[0, T] \ni t \mapsto x_{4}^{\mathrm{ref}}(t)$. This would have been a flatness-based implementation of an MPC controller. But here, it seemed more suitable to take advantage of years of practice of the operators and to mimic some of their reactions: we translated these in terms of the flat output via the flatness equations and ended up with an arbitrary choice of transition functions for the flat outputs. In the end we have a controller with a predictable behavior. It should be noted also that the computational effort required of the flatness based controller is extremely light compared to an MPC optimization-based technique: here the control is computed through two analytic expressions. Besides it

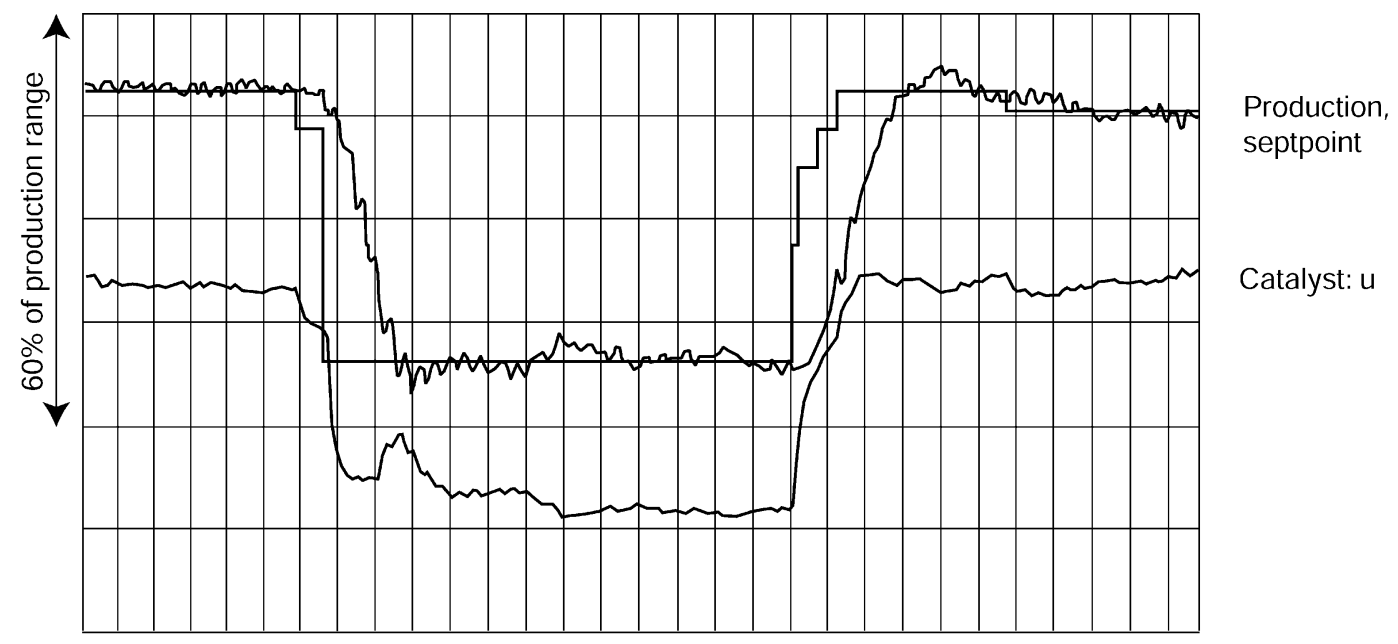

Fig. 4. Industrial results over 2 days (tests) with production (Prod) transients. The transients are fast and precise. Precise scales are omitted for confidentiality reasons. 


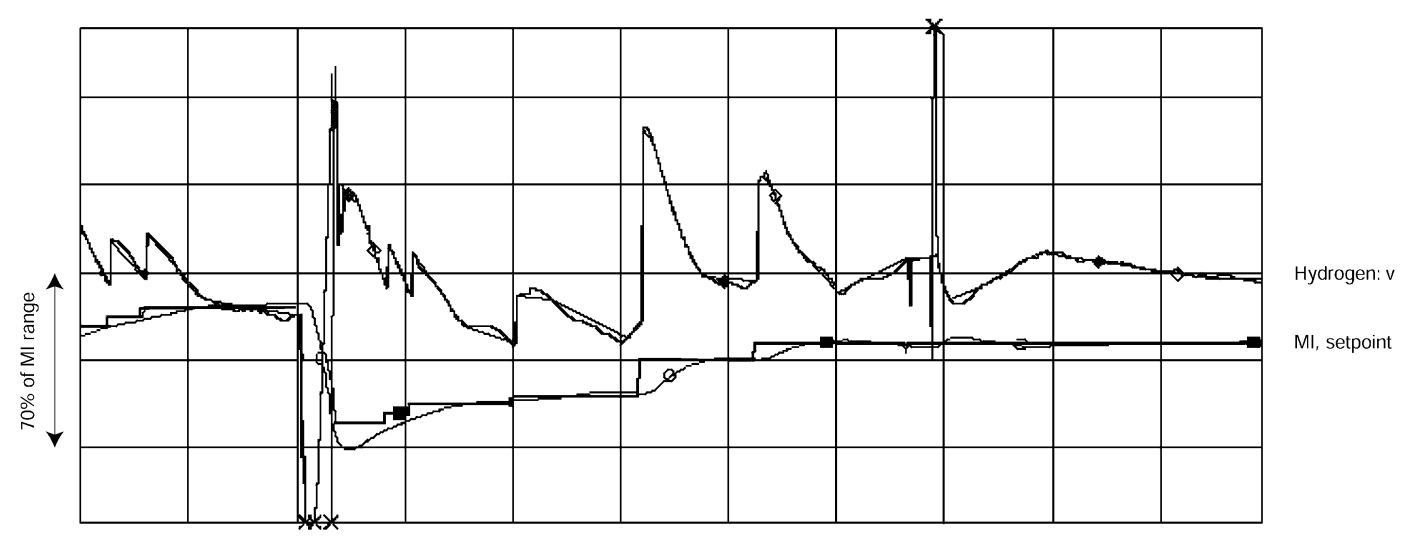

Fig. 5. Industrial results $10 \mathrm{~h}$ (tests) for melt-index $(M I)$ transients. The transients are fast and precise. Precise scales are omitted for confidentiality reasons. (The off-limit values are sensor failures.) The hydrogen actuator is much faster than the catalyst actuator, explaining the fast response of the system to a setpoint change.
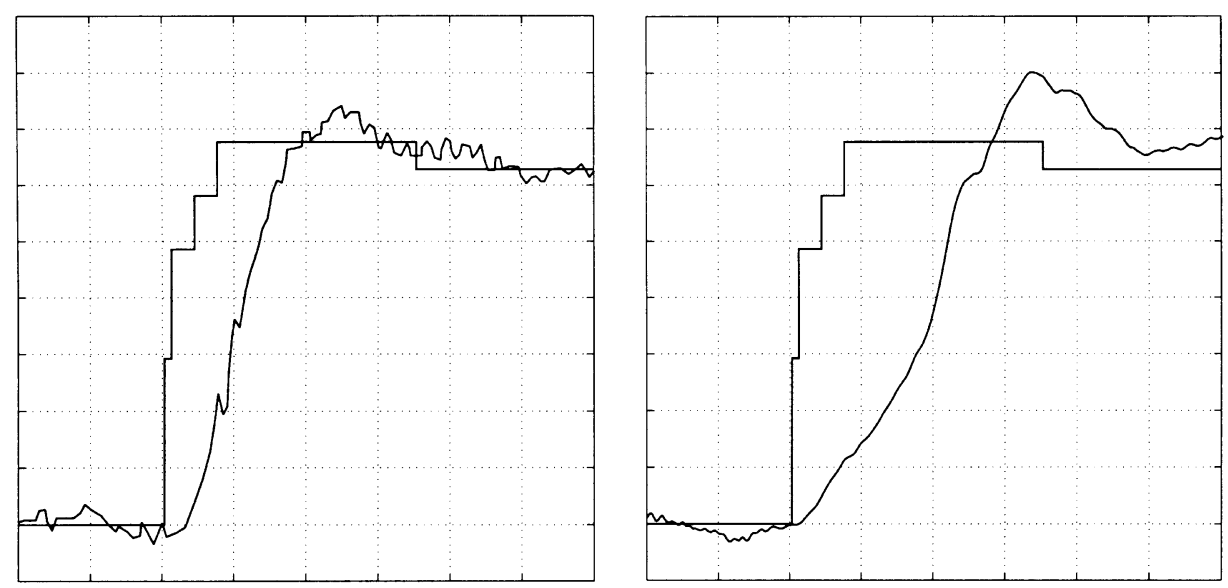

Fig. 6. Comparisons between our controller (test case real time results from history in Fig. 4 on the left) and a LQR controller for a simulation under similar conditions (right.) Prod transition. Time period $1 / 2$ day.

is possible to satisfy some min-max constraints on the inputs by saturating them without compromising the stability of the closed-loop system. On the other hand, this flatness based controller can not, as-is, handle stringent constraints of general forms. The MPC techniques would definitely be better suited for such cases.

\section{Conclusion}

The flatness of the system allows us to take into account the nonlinearities and the delay of the system. Though there is a tradeoff. On one hand we had to build a nonlinear model of the process which is time consuming and requires a good knowledge of the unit, on the other hand this allowed us to design an efficient controller in a relatively simple way. For such an accuracy demanding application we believe that this methodology is relevant and recommend it. A first order approximation of the unit would be less appropriate.
The key to our approach is the use of the flat outputs of the system. We found them easily thanks to the compactness of our model. It is true that there does not exist any "algorithm" to find the flat outputs. In the field of process control, at least, this is often not a big deal (see [13] for flat plug-flow reactor, flat mixing systems). As in mechanical engineering (see the flat pendulum [3]) the flat outputs always seem to have a strong physical meaning. In the present case this is also true: they are the rate of solid and the melt-index. Currently we are investigating different processes, trying to build relevant models and find their flat outputs.

More details about this particular application and other industrial control realizations in process control using flatness can be found in [13].

\section{References}

[1] S.K. Agrawal, N. Faiz, A new higher-order method for optimization of a class of nonlinear dynamic systems without Lagrange 
multipliers, Journal of Optimization Theory and Applications 97 (1) (1998) 11-28.

[2] B. Elvers, S. Hawkins, G. Schulz (Eds.), Ullmann's Encyclopedia of Industrial Chemistry. VCH, 1993.

[3] M. Fliess, J. Lévine, P. Martin, P. Rouchon, Flatness and defect of nonlinear systems: introductory theory and examples, Int. J. Control 61 (6) (1995) 1327-1361.

[4] M. Fliess, J. Lévine, P. Martin, P. Rouchon, A Lie-Bäcklund approach to equivalence and flatness of nonlinear systems, IEEE Trans. Automat. Control 44 (1999) 922-937.

[5] M. Fliess, H. Mounier, P. Rouchon, J. Rudolph. Systèmes linéaires sur les opérateurs de Mikusiński et commande d'une poutre flexible, in: ESAIM Proc. " Élasticité, viscolélasticité et contrôle optimal", 8ème entretiens du centre Jacques Cartier, Lyon, 1996, pp. $157-168$.

[6] R. Mahadevan, S. K. Agrawal, F. J. Doyle, Differential flatness based nonlinear predictive control of fed-batch bioreactors, in: IFAC Symposium on Advanced Control of Chemical Processes, 2000.

[7] P. Martin, R. M. Murray, P. Rouchon, Flat systems, in: Proc. of the 4th European Control Conf., 1997, pp. 211-264, Plenary lectures and Mini-courses.

[8] K.B. McAuley, J.F. MacGregor, On-line inference of polymer properties in an industrial polyethylene reactor, AIChE J. 37 (6) (1991) 825-835.
[9] K.B. McAuley, J.F. MacGregor, Optimal grade transitions in a gas phase polyethylene reactor, AIChE J. 38 (10) (1992) 1564 1576 .

[10] M.B. Milam, K. Mushambi, R.M. Murray, A new computational approach to real-time trajectory generation for constrained mechanical systems, in: IEEE Conference on Decision and Control, 2000, pp. 845-852.

[11] H. Mounier, Propriétés Structurelles des Systèmes Linéaires à Retards: Aspects Théoriques et pratiques, $\mathrm{PhD}$ thesis, Université Paris Sud, Orsay, 1995.

[12] H. Mounier, J. Rudolph, Flatness based control of nonlinear delay systems: a chemical reactor example, Int. J. Control 71 (1998) 871-890.

[13] N. Petit, Systèmes à Retards. Platitude en Génie des procédés et contrôle de Certaines Èquations des Ondes. PhD thesis, Ècole des Mines de Paris, 2000.

[14] N. Petit, Y. Creff, P. Rouchon, Minimum time constrained control of acid strength on a sulfuric acid alkylation unit, Chemical Engineering Science 56/8 (2001) 2767-2774.

[15] M. Roberson, APPRYL investments worth 1 billion french francs, Hydrocarbon Processing 77 (6) (June 1998).

[16] K. Wang, T. Loehl, M. Stobbe, S. Engell. A genetic algorithm for online scheduling of a mutiproduct polymer batch plant, in: 7th International Symposium on Process System Engineering, Computer and Chemical Engineering 24, 2000, pp. 393-400. 